\title{
ANALYSIS OF MAGNETIC ENERGY STORED IN \\ SUPERCONDUCTING COILS WITH AND WITHOUT FERROMAGNETIC \\ INSERTS*
}

by

Y. S. Cha

Materials and Components Technology Division

Argonne National Laboratory

9700 South Cass Avenue

Argonne, Illinois 60439

The submitted manuscript has been authored
by a contractor of the U. S. Government under
contract No. W-31-109-ENG-38. Accordingly,
the U. S. Government retains a nonexclusive,
royalty-free license to publish or reproduce the
publlshed form of this contribution, or allow
others to do so, for U. S. Government
purposes.

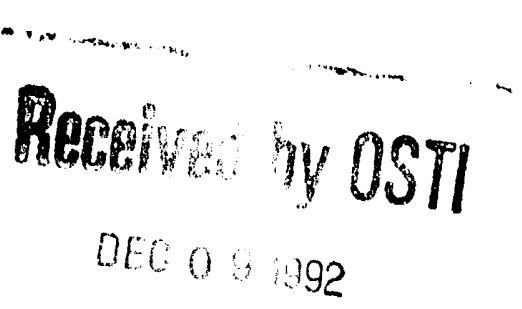

To be submitted to the 37th Annual Conference on Magnetism and Magnetic Materials, Houston, Texas, Dec. 1-4, 1992.

*Work at Argonne National Laboratory was performed under the auspices of the U.S. Department of Energy Superconductivity Pilot Center, Conservation and Renewable Energy, under Contract W-31-109-Eng-38.

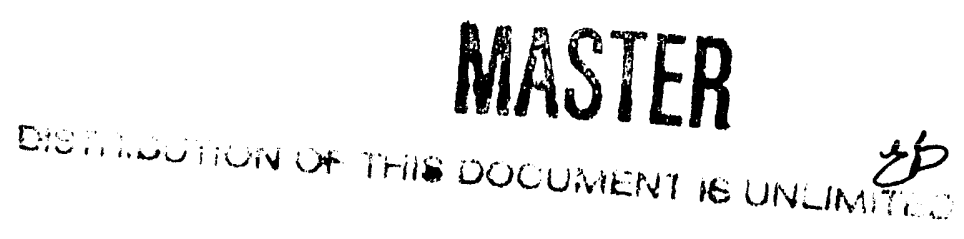




\title{
ANALYSIS OF MAGNETIC ENERGY STORED IN SUPERCONDUCTING COILS WITH AND WITHOUT FERROMAGNETIC INSERTS
}

\author{
Y. S. Cha
}

Materials and Components Technology Division

Argonne National Laboratory, Argonne, IL 60439

\begin{abstract}
Inductance and energy of superconducting coils are calculated by (1) a long solenoid approximation, (2) a finite element model, and (3) working formulas and tables. The results of the finite element model compare favorably with that of the working formulas. The long solenoid approximation overpredicts the energy and the inductance compared to the other two methods. The difference decreases with increasing length to diameter ratio.
\end{abstract}


Energy stored in a coil with a ferromagnetic insert are calculated by using the long solenoid approximation and the finite element model. The analysis shows that the gain in energy ratio is equal to the relative permeability of the insert. The relative permeability decreases with increasing current or current density. Even though large gains can be achieved at relatively low currents, the energy level itself is too low. The stored energy increases with current, but the gain decreases with increasing current because the relative permeability decreases. If a coil with a diameter of $0.3 \mathrm{~m}$ and a length of $0.3 \mathrm{~m}$ is required to store $10 \mathrm{~kJ}$ of energy, the current density must equal $4,000 \mathrm{~A} / \mathrm{cm}^{2}$. The gain in energy ratio is equal to 2.55 by using the insert.

Prediction of energy stored in a superconducting coil is essential to the design of superconducting magnetic energy storage systems. In this paper, we compare the results of three different methods of calculating the energy stored in superconducting coils. These methods are: (1) long solenoid approximation, (2) finite element analysis, and (3) working formulas and tables for inductances. Our primary objective is to calculate the energy stored in a solenoid coil with a ferromagnetic insert and to determine the gain in energy compared to a coil without insert. The reference coil has a diameter of $0.30 \mathrm{~m}$, an axial length of 0.30 $\mathrm{m}$, and a thickness of $2 \mathrm{~cm}$.

If the length is large compared with the diameter of the coil, the following equations are expected to apply

$$
\begin{aligned}
& \mathrm{U}_{0}=\varepsilon_{0} \mathrm{C}^{2} \mathrm{~B}_{0}^{2}\left(\pi \mathrm{R}^{2}\right) \ell / 2, \\
& \mathrm{~B}_{0}=\frac{\mathrm{Ni}}{\varepsilon_{0} \mathrm{C}^{2} \ell}
\end{aligned}
$$


where $U_{0}=$ magnetic energ $y, \varepsilon_{0} C^{2}=10^{7} / 4 \pi, B_{0}=$ magnetic flux density, $N=$ total number of turns of the winding, $i=$ current, $R=$ radius of coil, and $\ell=$ length. The inductance of the coil (L) is obtained by letting

$$
\mathrm{U}_{0}=\frac{1}{2} \mathrm{Li}^{2},
$$

which results in

$$
L=\pi R^{2} N^{2} / \varepsilon_{0} C^{2} \ell .
$$

The finite element program used in this report is PE2D which is developed by Vector Fields. ${ }^{1}$ The calculations are performed by using the axisymmetric option in the code. The energy stored is calculated by integrating over the entire computational domain. It should It should be noted that PE2D does not provide inductance of the coil. However, the inductance can be calculated by using the energy obtained from PE2D. Equation 3 can be rewritten as

$$
\mathrm{U}_{0}=\frac{1}{2} \mathrm{Li}^{2}=\frac{1}{2} \mathrm{~L}\left(\frac{\mathrm{I}}{\mathrm{N}}\right)^{2},
$$

where $I(=\mathrm{Ni})$ is the total current over the entire cross-sectional area of the winding. The inductance is

$$
\mathrm{L}=\frac{2 \mathrm{U}_{0} \mathrm{~N}^{2}}{\mathrm{I}^{2}} .
$$


The total current I is the product of the current density and the total crosssectional area of the winding.

The inductance (and hence, energy) of coils can be calculated by the following working formula developed by Grover ${ }^{2}$

$$
\mathrm{L}=0.019739\left(\frac{2 \mathrm{R}}{\ell}\right) \mathrm{RN}^{2} \mathrm{~K}^{\prime}
$$

where $\mathrm{K}^{\prime}$ is a function of the geometrical dimension of the coil and can be obtained from the tables provided in Ref. 2. Note that Eqs. 4 and 7 are similar and both indicate that the inductance is proportional to $\mathrm{R}^{2} \mathrm{~N}^{2}$ and inversely proportional to $\ell$. The stored energy is calculated by using Eq. 3.

Table 1 shows the comparison of the inductance $\left(L / N^{2}\right)$ as a function of $\ell / D$ calculated by using the three methods. The values listed in Table 1 are equal to $\mathrm{L} / \mathrm{N}^{2}$. This is because the inductance $\mathrm{L}$ is proportional to $\mathrm{N}^{2}$ for all three methods (Eqs. 4, 6, and 7). The actual values of $L$ can be obtained by multiplying the values in Table 1 by $\mathrm{N}^{2}$. The inductance calculated by PE2D agrees very well with that calculated by working formulas. The inductance calculated by the long solenoid approximation is always greater than that of either the PE2D or the working formula. The difference decreases with increasing $\ell / D$.

It should be mentioned that the finite element analysis provides not only the energy stored, but also the spatial distribution of the magnetic flux density. The latter cannot be obtained from the other two methods. The magnetic flux density distributions along radial directions at two vertical elevations for the reference coil with a current density of $1,000 \mathrm{~A} / \mathrm{cm}^{2}$ are shown in Figs. 1 and 2. At $\mathrm{z}=0$ (center of the coil), the vertical component $\left(B_{z}\right)$ of the flux density is fairly uniform inside the coil and it drops sharply across the thickness of the coil. The radial 
component $\left(B_{r}\right)$ of the flux density is always small at this elevation. At $z=0.15 \mathrm{~m}$ (upper end of the coil), $B_{z}$ is fairly uniform inside the coil, but there is also a large $B_{r}$ everywhere in the coil. $B_{r}$ reaches its maximum at the coil itself. Figure 3 shows the distribution of the magnitude of the flux density at $z=0.15 \mathrm{~m}$. The maximum of $\mathrm{B}$ at this elevation is approximately 0.17 Tesla and occurs at the upper edge of the coil. However, this maximum is still smaller than the vertical component $\left(B_{z}\right)$ of the flux density anywhere inside the coil at $z=0$. The flux density calculated by using the long solenoid approximation, Eq. 2, is equal to 0.25 Tesla which is larger than that calculated by PE2D anywhere in the coil.

The energy stored in a coil with an insert (U) can be approximated by

$$
\mathrm{U}=\varepsilon_{0} \mathrm{C}^{2}\left(\pi \mathrm{R}^{2} \ell\right) \mu \mathrm{H}^{2} / 2
$$

where $\mathrm{H}$ is the applied field strength and the relative permeability $\mu$ is defined as

$$
\mu=\mathrm{B} / \mathrm{H}
$$

Dividing Eq. 8 by Eq. 1 gives

$$
\frac{\mathrm{U}}{\mathrm{U}_{0}}=\mu \mathrm{H}^{2} / \mathrm{B}_{0}^{2}=\mu
$$

because $\mathrm{H}$ is the magnetizing field which should be equal to the magnetic flux density in a material with zero magnetization, i.e., the magnetic flux density of a coil with an air core $B_{0}$. Thus, the gain in stored energy by using an insert is equal to the relative permeability $\mu$ which is also the gain in magnetic flux density $\mathrm{B}$ due to the magnetizing field $\mathrm{H}$ (Eq. 9). Different materials have different B-H curves and thus, different values of $\mu$. Furthermore, B-H curves are usually 
nonlinear and the value of $\mu$ can vary over a wide range and depends strongly on the magnetizing current (or field). The key issue here is to determine the energy stored (and the gain) in a coil with insert as a function of current density.

As a specific example, we choose METGLAS Alloy $2605 \mathrm{C} 0$ as the insert material. This material has relatively high saturation flux density $B_{S}$ of $\sim 1.7$ Tesla. The B-H curve of METGLAS $2605 \mathrm{CO}$ is shown in Fig. 4. The B-H curve in Fig. 4 can be conveniently divided into two regions if a saturation magnetizing field $\mathrm{H}_{\mathrm{S}}$ is defined as the $\mathrm{H}$ corresponding to $\mathrm{B}_{\mathrm{S}}$. As shown in Fig. 4, $\mathrm{B}_{\mathrm{S}}=1.7$ Tesla and $\mathrm{H}_{\mathrm{S}}=0.025$ Oersteds. For $\mathrm{H}<\mathrm{H}_{\mathrm{S}}$, the B-H curve rises sharply. For $\mathrm{H} \geq$ $\mathrm{H}_{\mathrm{S}}$ further increase in $\mathrm{H}$ will not cause additional magnetization. In this region, $B$ becomes proportional to $\mathrm{H}$ and with a unit slope. Thus, for $\mathrm{H} \geq \mathrm{H}_{\mathrm{S}}$, we can use the following approximation

$$
B=B_{S}+\frac{d B}{d H} \Delta H=B_{S}+H-H_{S}
$$

and

$$
\mu=\frac{B}{H}=\frac{B_{S}+H-H_{S}}{H} .
$$

In the limit of $\mathrm{H}>>\mathrm{B}_{\mathrm{S}}>\mathrm{H}_{\mathrm{S}}, \mu=1$, and there is no gain in either the stored energy or the magnetic flux density. Figure 4 also shows the variation of the relative permeability $\mu$ with the magnetizing field $H$. At small $H, \mu$ is high and increases with $\mathrm{H}$. As $\mathrm{H}$ is increased slightly beyond $\mathrm{H}_{\mathrm{S}}, \mu$ begins to decrease sharply and approaches unity at very large $\mathrm{H}$.

Our interest is the energy stored in a coil with insert and the results are shown in Fig. 5. Also shown in this figure is the energy ratio $U / U_{0}$, where $U$ and 
$\mathrm{U}_{0}$ are the energy stored in a coil with and without insert, respectively. Large gains can be achieved at relatively low current densities, but the energy level is too small. The stored energy increases with current density but the gain decreases with current density. For example, if a coil with a diameter of $0.3 \mathrm{~m}$ and a length of $0.3 \mathrm{~m}$ is required to store $10 \mathrm{~kJ}$ of energy, the current density must equal 4,000 $\mathrm{A} / \mathrm{cm}^{2}$ and the gain is 2.55 by using an insert.

Finite element calculations for coil with an insert is also carried out by using PE2D. Figure 6 shows the comparison of the stored energy for the reference coil with insert calculated by using PE2D and the long solenoid approximation. As expected, the long solenoid approximation always overpredicts the energy stored in a coil because the length-to-diameter ratio for the reference coil is not long enough to make the results of the long solenoid approximation approach that of PE2D.

Work at Argonne National Laboratory was performed under the auspices of the U.S. Department of Energy Superconductivity Pilot Center, Conservation and Renewable Energy, under Contract W-31-109-Eng-38. 


\section{References}

1. PE2D, Vector Fields, LTD., 24 Bankside, Kidlington, Oxford OX-5 1JE, England.

2. F. W. Grover, Inductance Calculations, Working Formulas and Tables, Dover Publ., Inc., Chapter 13 (1946). 
Table 1. Comparison of Inductance ( $\left.L / N^{2}\right)$ Calculated by the Three Methods $(\mu H)$

\begin{tabular}{|c|c|c|r}
\hline$\frac{\ell}{D}$ & Long Solenoid & PE2D & Working \\
\hline 0.333 & 0.887 & 0.348 & 0.3 \\
0.666 & 0.443 & 0.247 & 0.2 \\
1.000 & 0.295 & 0.192 & $0.1 \%$ \\
1.666 & 0.177 & 0.133 & $0.1 \%$ \\
3.333 & 0.088 & 0.075 & 0.0 \\
\hline
\end{tabular}




\section{FIGURE CAPTIONS}

Fig. 1. Distributions of vertical and radial components of magnetic flux density calculated by PE2D $(z=0)$

Fig. 2. Distributions of vertical and radial components of magnetic flux density calculated by PE2D $(z=0.15 \mathrm{~m})$

Fig. 3. Distribution of the magnitude of magnetic flux density calculated by PE2D $(z=0.15 \mathrm{~m})$

Fig. 4. Variations of relative permeability and magnetic flux density with magnetic field strength for METGLAS 2605C0

Fig. 5. Variations of $U / U_{0}$ and $U$ with current density calculated by using the long solenoid approximation

Fig. 6. Comparison of stored energy versus current density calculated by using the long solenoid approximation and finite element model 


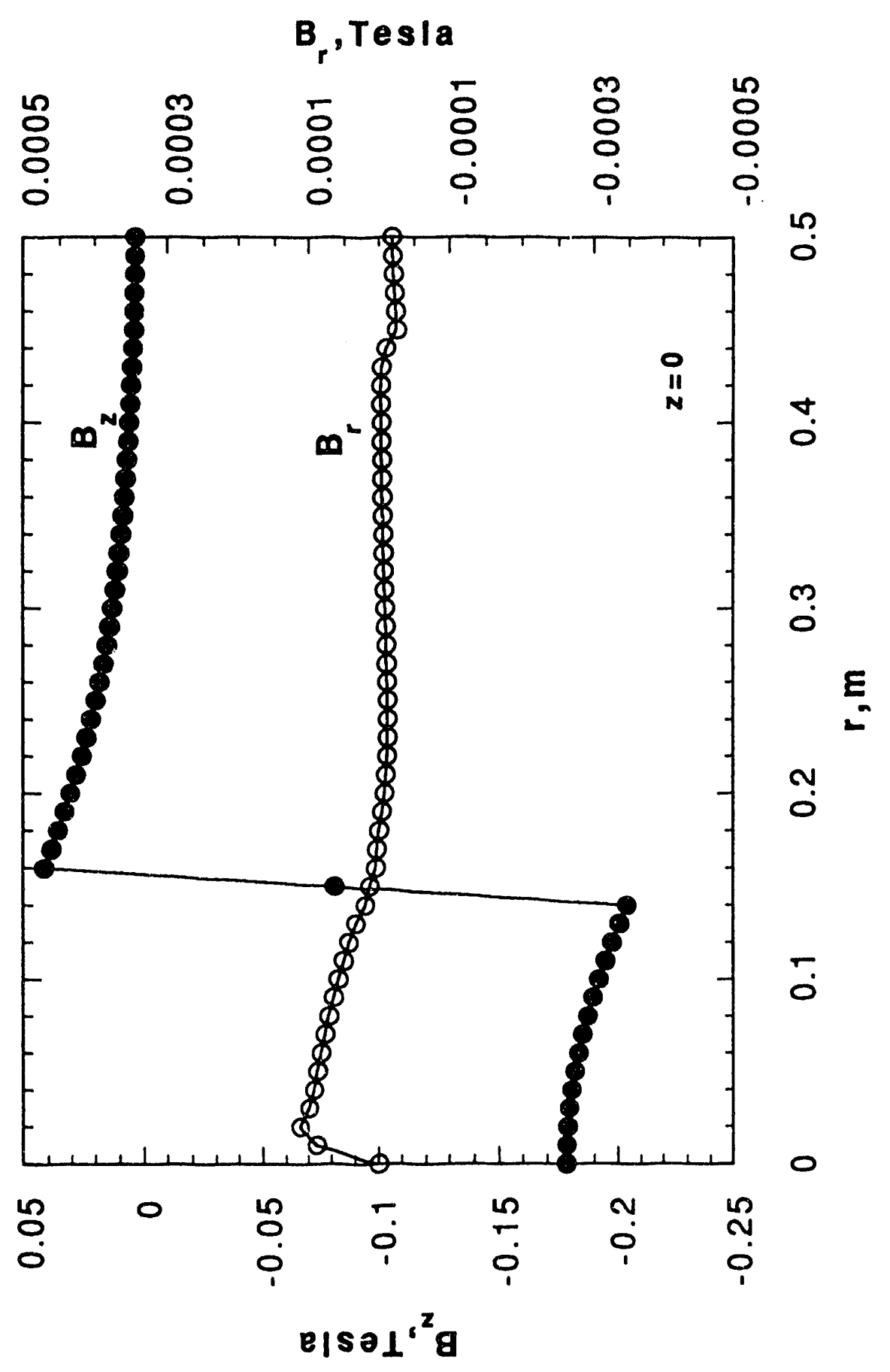



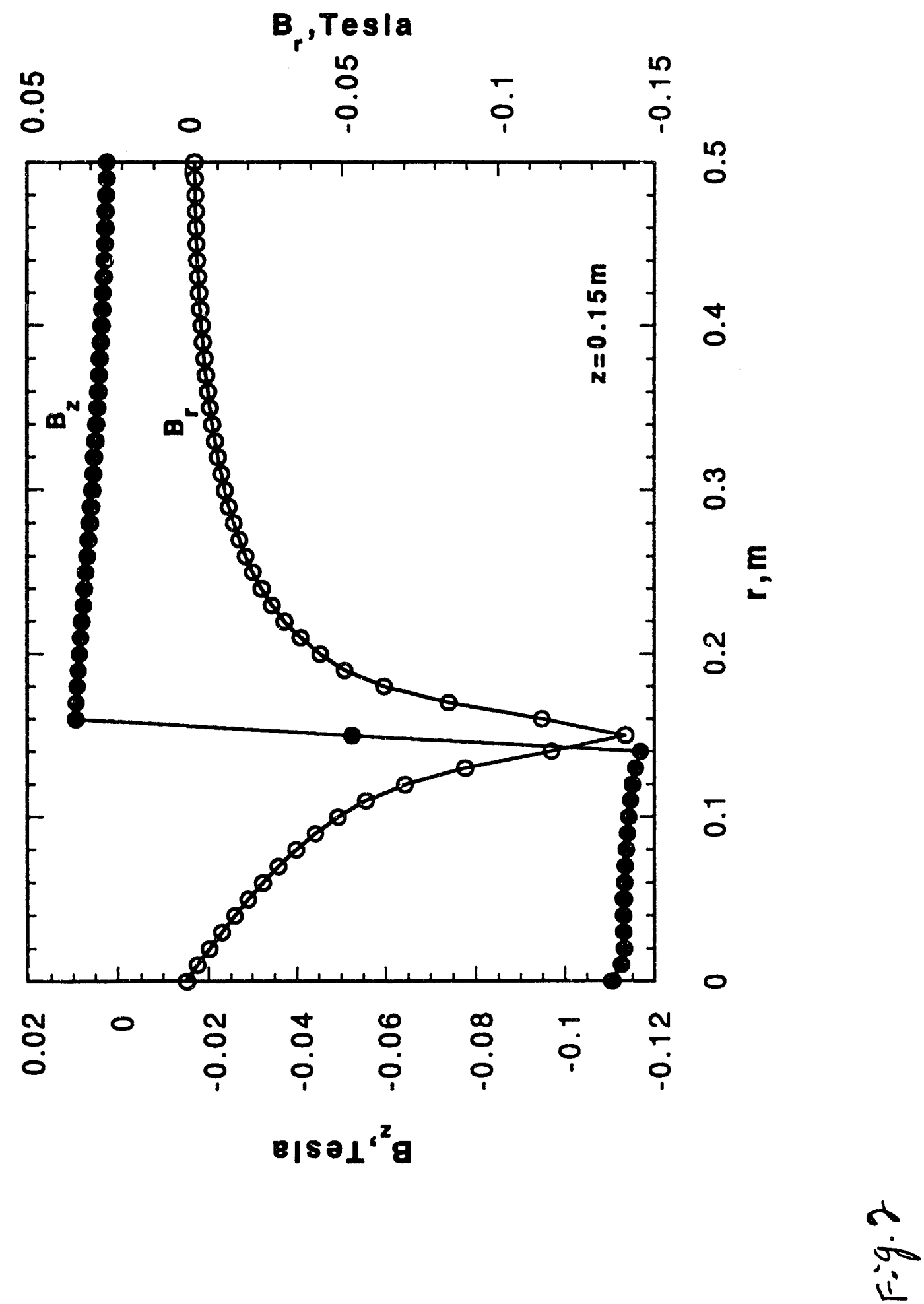


$$
I
$$




\section{Permeability}

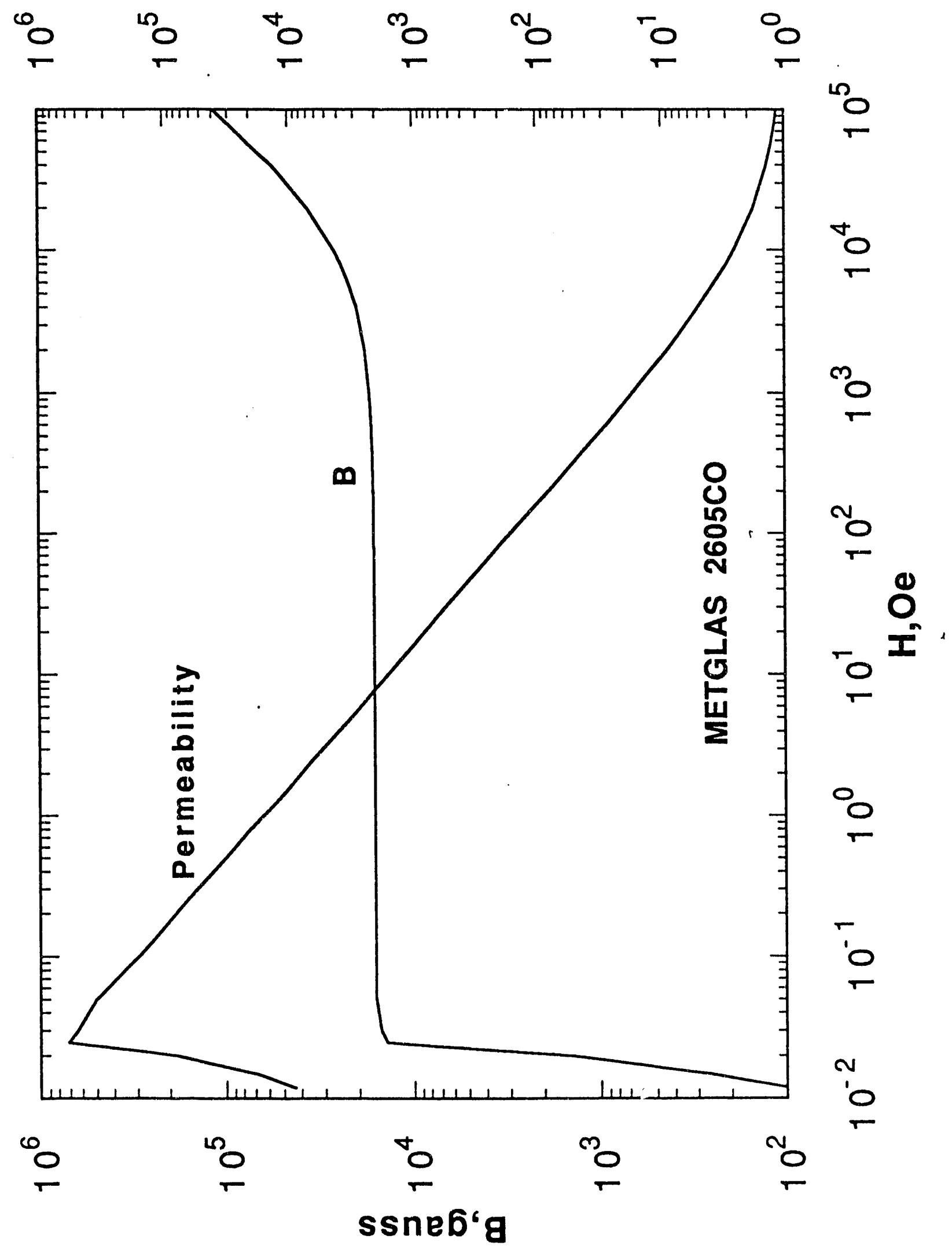




\section{Energy(U),kJ}

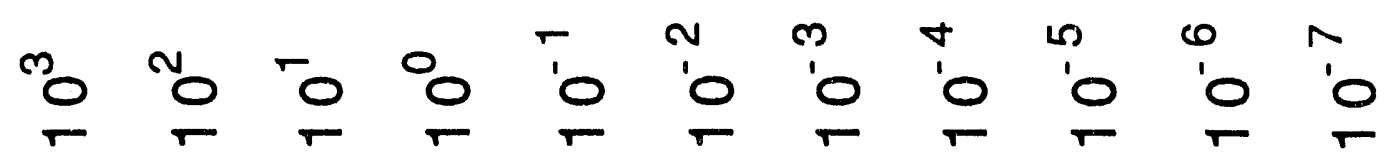

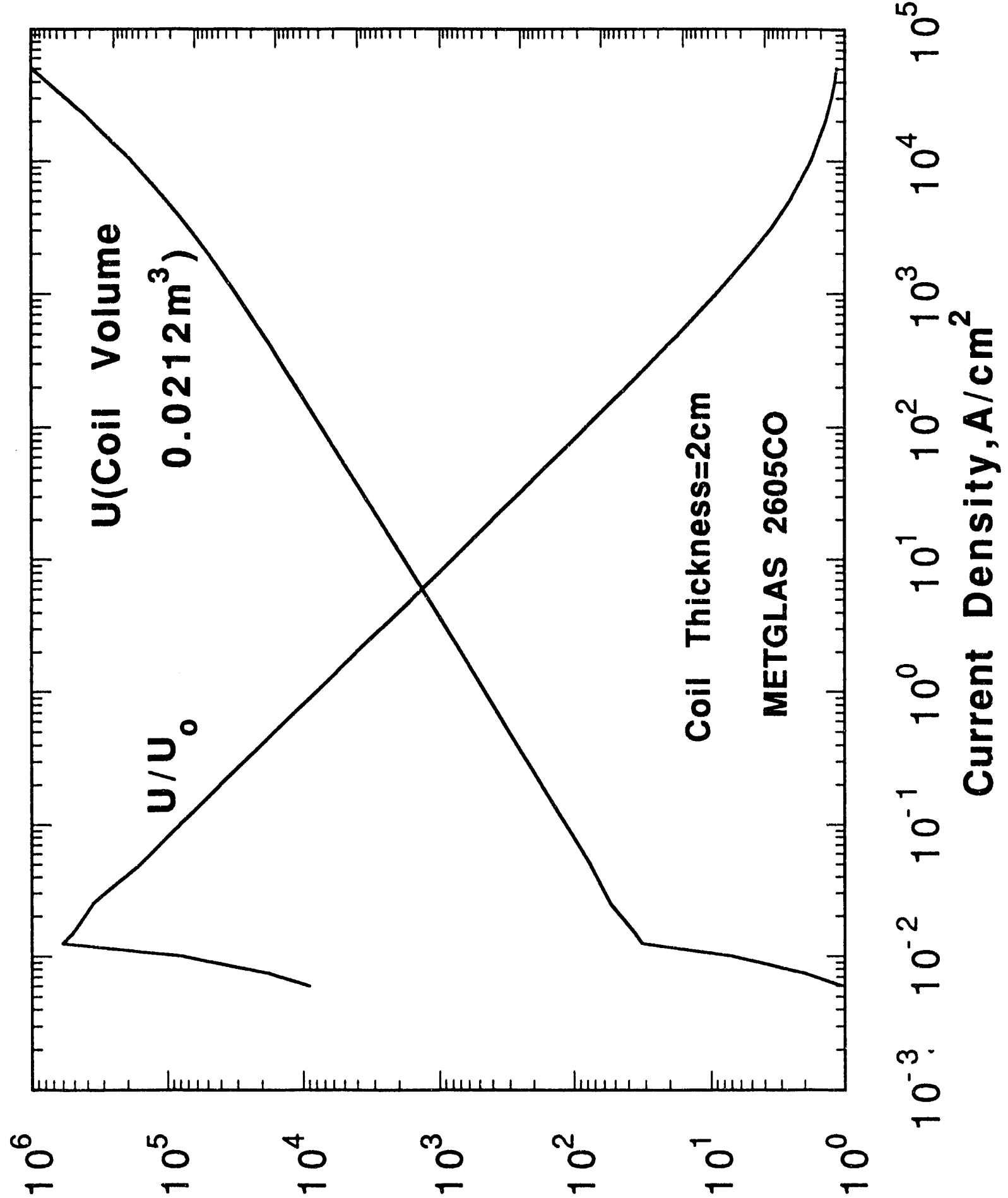

o 


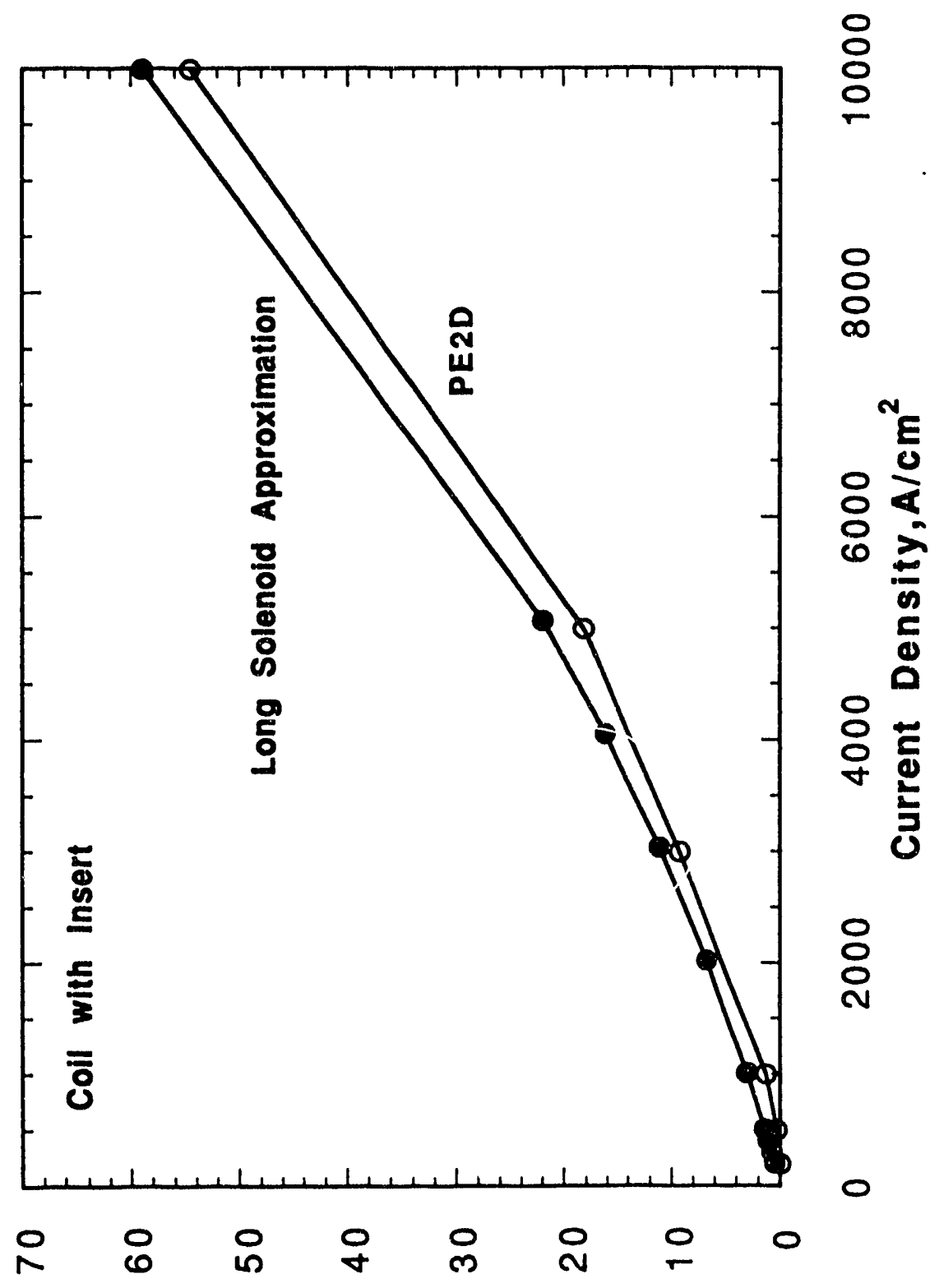

ry'K6,aug padols 

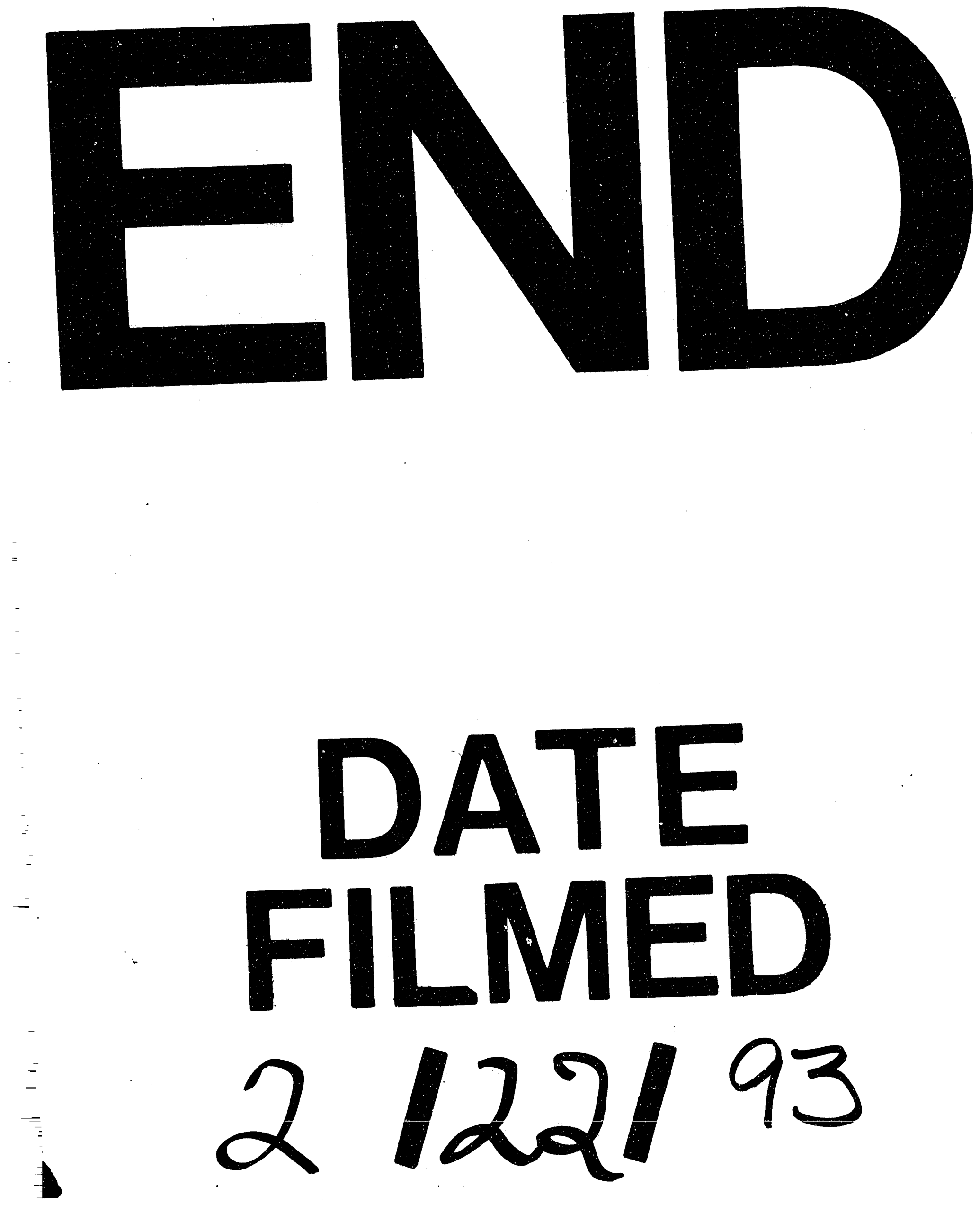
